\title{
The future of medical education: areas that should receive increased focus in the 21st Century
}

\author{
Michele Heisler
}

$\mathrm{W}$ hat is fundamental for medical students to learn in the $21^{\text {st }}$ Century? Many medical educators understandably focus on the explosion of new medical knowledge and technology that will be essential for medical students to master in the $21^{\text {st }}$ Century. These include advances in molecular medicine, genomics, a plethora of new pharmacological approaches, among other stunning discoveries in medicine. Moreover, there will be a continued need to ensure that medical students acquire the highest level of professional ethics, leadership skills, empathy, and communication and patient-centered counseling skills. All of these have been a core focus of medical educators over the past decade and will continue to be a priority. In thinking about the $21^{\text {st }}$ Century, there are several key additional areas of content knowledge and skills that will be essential for future physicians that to date have not received sufficient attention in medical skill curricula. These include: 1) a deep understanding of the social determinants of health and approaches to screen for and address these; 2) approaches to 'personalized medicine' to tailor treatments both to patients' clinical profiles and to their preferences, values, and life circumstances; 3 ) working effectively in interdisciplinary teams; and 4) making effective use of Information Technology to improve clinic visits, between face-to-face visit outreach, and proactive identification of and assistance to meet both individual and population health needs.

To date, too little attention has been devoted in most medical curricula to what are called the 'social determinants of health'. Inequalities in the social determinants of health, defined as the 'economic, environmental, political, and social conditions in which people are born, live, and age', contribute to a disproportionate burden of disease borne by socially disadvantaged groups. To enhance the health of patients with complex social needs, it is essential to screen for and help address the social and environmental barriers to health they face, coordinate and manage care between medical visits, and create effective links between health care and community resources. Future physicians will need to have a more robust understanding of the sources of health inequities that exist within and between populations, of the types of interdisciplinary collaborations and most effective strategies necessary to address these. This will require training and practical experiences in population health, in social as well as clinical epidemiology, and in program design and evaluation.

In order to better meet the medical and social needs of patients, medical students will need to be well trained in innovative approaches to 'Personalized Medicine'. Personalized medicine has been defined as a 'medical

MD, MPA, Professor of Internal Medicine and Health Behavior and Health Education; Co-Director, Robert Wood Johnson Foundation Clinical Scholars ${ }^{\circledR}$; Associate Director, Global REACH, University of Michigan, Research Scientist, Center for Clinical Management Research, Ann Arbor VA HSR\&D. 
model that separates patients into different groups with medical decisions, practices, interventions and/or products being tailored to the individual patient based on their predicted response or risk of disease'. To be truly effective and patient-centered, medical educators need to ensure that medical students adopt a broader understanding of personalized medicine beyond this narrow definition. Key to personalized medicine is ensuring that the right patient receives the right treatment at the right time. The 'right' treatment, however, also means that the treatment meets the individual patient's own preferences and values. Physicians need to have effective skills to engage in shared decision-making with patients, to elicit what is most important to them, and what best meets their needs and life circumstances. As the populations in countries like Brazil and the United States age and live with multiple chronic conditions, physicians will have to have the medical knowledge to prioritize among multiple conditions as well as the communication skills to discuss multiple options with patients. Treatment options continue to expand, and many of these treatments have similar clinical outcomes but with different costs and benefits. Accordingly, physicians will need to communicate clearly to their patients about the trade-offs involved with different treatment options and work with them to determine the best treatment tailored to their individual needs, preferences, and conditions.

In recent years, increased attention has been given in medical education to preparing medical students to practice in an environment in which much care has moved from inpatient to outpatient environments. Another key transformation in care has been the emergence of teambased care. Interdisciplinary teams are an efficient and effective way to provide coordinated, integrated care that addresses the multiple needs of increasingly complex patients. Such teams may range from 'teamlet' models of a physician, a nurse, and a medical assistant, to those in Brazil's Family Health Program of a physician, a nurse, a medical assistant, and a community health agent, to those in the United States's Veterans Affairs (VA) health systems with physicians, nurses, clinical pharmacists, social workers, and behavioral health specialists. To meet the potential of team-based care, trainees need to develop the skills and understanding to be able to work effectively in such interdisciplinary teams. To do this, training programs will need to create more opportunities for trainees from different disciplines to participate in joint training activities, practice working together in teams, and thereby cultivate the requisite sensitivity and collaboration skills to prepare them for their future team roles.

A key aim of medical school curricular reforms over the past decades has been to inculcate in medical students habits and skills necessary for lifelong learning and problem-solving. Medical schools have emphasized 'problem-based learning' in which small groups of students work together with the guidance of a faculty member to solve difficult patient cases. In light of the exponential growth of new medical discoveries and vast amount of rapidly changing medical knowledge, an increasing priority has been to train students in the use of online resources to formulate appropriate clinical questions and efficiently answer them. In the $21^{\text {st }}$ Century, medical students will need to master a much wider array of online resources and Information Technology (IT) tools and learn to effectively integrate them into all areas of their clinical practice. They will need to learn to use such resources to prepare for clinic visits with individual patients, to use interactively during clinic visits to share information with and assist in shared decision-making with patients, and to communicate with and extend support to patients between face-to-face office visits through patient portals, email, text messages, and other m-health and e-health tools. Beyond use with individual patients, IT tools will allow for more efficient population management and proactive identification of and outreach to vulnerable patients in need of specific types of assistance. Geographic information systems (GIS) and Web technologies, for example, will be more effectively deployed to better link inpatient and outpatient care, identify communities with especially great health needs and/or poor quality of health care, and allow for more effective medical and public health approaches to address both medical and social determinants of health. Medical students will need training and practice to learn to seamlessly use current IT approaches and be prepared to continue incorporating new IT advances into their practice. 